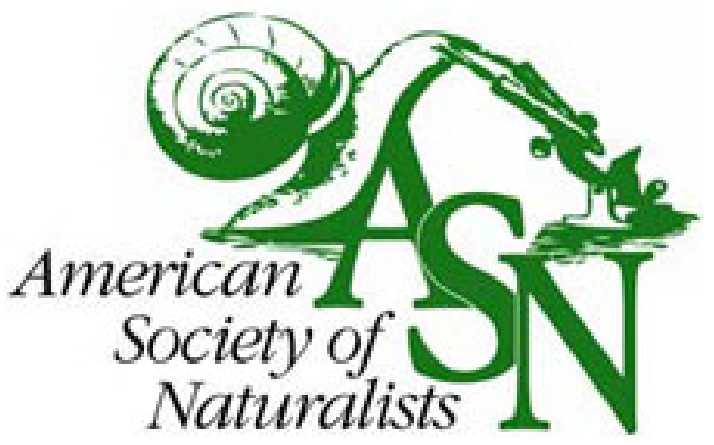

Identifying the Critical Climatic Time Window That Affects Trait Expression. Author(s): Martijn van de Pol and Andrew Cockburn

Source: The American Naturalist, Vol. 177, No. 5 (May 2011), pp. 698-707

Published by: The University of Chicago Press for The American Society of Naturalists

Stable URL: http://www.jstor.org/stable/10.1086/659101

Accessed: 28/04/2011 23:09

Your use of the JSTOR archive indicates your acceptance of JSTOR's Terms and Conditions of Use, available at http://www.jstor.org/page/info/about/policies/terms.jsp. JSTOR's Terms and Conditions of Use provides, in part, that unless you have obtained prior permission, you may not download an entire issue of a journal or multiple copies of articles, and you may use content in the JSTOR archive only for your personal, non-commercial use.

Please contact the publisher regarding any further use of this work. Publisher contact information may be obtained at http://www.jstor.org/action/showPublisher?publisherCode=ucpress.

Each copy of any part of a JSTOR transmission must contain the same copyright notice that appears on the screen or printed page of such transmission.

JSTOR is a not-for-profit service that helps scholars, researchers, and students discover, use, and build upon a wide range of content in a trusted digital archive. We use information technology and tools to increase productivity and facilitate new forms of scholarship. For more information about JSTOR, please contact support@ jstor.org. 


\title{
Identifying the Critical Climatic Time Window That Affects Trait Expression
}

\author{
Martijn van de Pol${ }^{\star}$ and Andrew Cockburn
}

Evolution, Ecology and Genetics, Research School of Biology, Australian National University, Canberra, Australian Capital Territory 0200, Australia

Submitted October 27, 2010; Accepted January 7, 2011; Electronically published March 31, 2011

Online enhancement: zip file. Dryad data: http://datadryad.org/handle/10255/dryad.8277.

ABSTRACT: Identifying the critical time window during which climatic drivers affect the expression of phenological, behavioral, and demographic traits is crucial for predicting the impact of climate change on trait and population dynamics. Two widely used associative methods exist to identify critical climatic periods: slidingwindow models and recursive operators in which the memory of past weather fades over time. Both approaches have different strong points, which we combine here into a single method. Our method uses flexible functions to differentially weight past weather, which can reflect competing hypotheses about time lags and the relative importance of recent and past weather for trait expression. Using a 22-year data set, we illustrate that the climatic window identified by our new method explains more of the phenological variation in a sexually selected trait than existing approaches. Our new method thus helps to better identify the critical time window and the causes of trait response to environmental variability.

Keywords: climate change, method, phenology, sliding window, timeto-event models, weather package.

\section{Introduction}

The expression of many traits varies over time because of differences in environmental conditions. Understanding how traits respond to climatic variation is of key interest to both evolutionary biologists and ecologists (Ozgul et al. 2009). For example, the response of phenological traits - such as timing of breeding - to climatic conditions will determine whether species will have sufficient phenotypic plasticity to adapt to climate change or whether their survival depends on an evolutionary response (Charmantier et al. 2008; Visser 2008). The response of demographic traits (reproduction and survival) to climate variability will also determine whether populations decline or increase under future climate change (Hallet et al. 2004; van de Pol et al. 2010). We thus need to be able to identify the critical time window during which climatic drivers affect the expression of traits to predict the future impact

* Corresponding author; e-mail: m.van.de.pol@myscience.eu.

Am. Nat. 2011. Vol. 177, pp. 698-707. (C) 2011 by The University of Chicago. 0003-0147/2011/17705-52579\$15.00. All rights reserved. DOI: $10.1086 / 659101$ of climate change and variability on trait dynamics and on population dynamics. A difficulty, however, is that the environment could be related to a trait in many different ways, since organisms are responding to a history of multidimensional environmental variation.

Models that try to identify critical climatic windows can be either mechanistic or associative. Mechanistic models aim to include information about the underlying biological processes that determine the timing or degree of trait expression (Trudgill et al. 2005). For example, hydrothermal time models use temperature- and soil-water-dependent development rates of seeds to predict emergence rates (Izquierdo et al. 2009). Mechanistic models require detailed biological data about the processes involved in trait expression, which can generally only be obtained by controlled experiments. Consequently, mechanistic models have primarily been used for commercially interesting species (crops, fruits, and forestry) and are generally not a feasible option for ecological studies on wild animals.

Associative models are not directly based on any particular biological mechanism but rather examine quantitative associations between weather variables and trait expression. As in most associative approaches, the underlying idea is that the climatic time window that best explains or predicts the observed variation in trait expression is likely to have a causal role. By far the most popular associative method is the sliding-window approach (e.g., Hallett et al. 2004; Estrella et al. 2007; Brommer et al. 2008; Husby et al. 2010). In this method, one calculates the mean of a weather variable over a given time period (e.g., January-March temperature) and then determines the strength of association (i.e., cross-correlation) with the dependent variable of interest. By varying the starting date_reflecting time lags_-and the width of the sliding time window-reflecting the duration of the windowone aims to identify the critical climatic period that affects trait expression (i.e., the window producing the highest cross-correlation).

Akin to the sliding-window method is the multiple regression approach (e.g., Fitter et al. 1995; Sparks and Carey 1995; Roberts 2008). In the multiple regression approach, 
aggregates of climate variables_-such as monthly mean temperatures-are entered simultaneously as separate predictors in a multiple regression analysis. If, for example, the separate mean temperatures of January, February, and March are the only predictors retained in the final model, we could conclude that the period January-March is the critical time window affecting trait expression. Since multiple regression approaches typically identify contiguous critical climatic time windows (e.g., Fitter et al. 1995; Sparks and Carey 1995), the multiple regression approach is conceptually similar to the sliding-window method and henceforth not considered separately.

The sliding-window approach clearly has merit: it is simple, reflects the basic characteristics of a critical time window, and allows for time lags. However, there are some limitations. First, the method typically uses arbitrary cutoff points for time periods-usually the first or last day of a month - which may not be biologically realistic (but see Roberts 2008). Second, the method assumes that all days included in the critical time window exert equal influence on trait expression.

To overcome some of the above limitations, Gienapp et al. (2005; see also Bauer et al. 2008; Gienapp et al. 2010) suggested a different associative method that does not focus on aggregated time periods, such as monthly means, and also weights past weather differently from more recent weather. Specifically, Gienapp and coworkers used the Bush-Mosteller linear operator (Bush and Mosteller 1955), which classically has been used in learning models:

$$
I_{t}=(1-\alpha) c_{t}+\alpha I_{t-1} .
$$

In this recursive equation, the weather index $I$ is iteratively updated each time step $t$ as a function of the value of the weather variable of interest $c$ and the value of index $I$ in the previous time step. The weighting parameter $\alpha$ determines how strongly the past is "remembered" compared to the present.

Although the Bush-Mosteller approach avoids the problem that all days within the window are weighted evenly, the method produces only indices in which the most recent weather is weighted more strongly than past weather. It therefore fails to allow for time lags in the response of the trait to weather (see also "Material and Methods"). Time lags are expected in biological systems for many reasons, including cases in which $(a)$ the physiological response involves a maturation period (e.g., egg or seed production) or $(b)$ the effect of weather is mediated via growth or reproduction of a food source (e.g., initiation of breeding, departure for migration). In addition, the Bush-Mosteller approach only allows for less-than-proportional decreases in the importance of past weather over time, while an accelerating decrease over time-where the memory of past weather fades more rapidly — may also be biologically plausible.

Here we combine the strong points of the slidingwindow approach as well as the Bush-Mosteller approach into a single new method, which allows for direct testing of competing hypotheses about time lags and the relative importance of recent and past weather for trait expression. Our method uses a flexible class of weighting functions derived from the family of extreme value probability distribution functions. These weighting functions have three strategic parameters that allow critical climatic windows to vary in shape, width, and location, which can be optimized using maximum likelihood methods. We illustrate our simple and intuitive method with a 22-year data set on a sexually selected phenological trait (timing of prenuptial molt) in superb fairy wrens (Malurus cyaneus).

\section{Material and Methods}

Framework of the New Method

Like existing associative methods, we aim to generate a univariate weather index that can be regressed against trait expression data using any type of regression model (generalized linear [mixed] models, Cox-type regressions, etc.). In our new method, we choose index $I$ to be a weighted average of the climatic variable of interest $c$ over the period $p$ preceding the measurement of trait expression:

$$
I_{t}=\sum_{j=0}^{j=p} w_{j} \times c_{t-j} .
$$

Before introducing the weight functions $w_{j}$ used in our new method, for comparative reasons we first show that it is possible to rewrite both the sliding-windows and BushMosteller approaches in terms of weight functions.

For a sliding window with starting point $\tau \geq 0$ and window width $\psi>0$, the weight at $j$ time steps in the past is (see also fig. 1a):

$$
w_{j}=\left\{\begin{array}{ll}
1 / \psi & \text { if } j \geq \tau \text { and } j<\tau+\psi \\
0 & \text { if } j<\tau \text { or } j \geq \tau+\psi
\end{array} .\right.
$$

For the Bush-Mosteller approach (eq. [1]), the weight function is effectively equivalent to an exponential decay function (see app. A):

$$
w_{j}=\alpha^{j},
$$

where $\alpha \in\langle 0,1]$. If $\alpha$ is close to 0 , the weather index equals the most recent weather, while all time intervals are weighted virtually equally when $\alpha$ is close to 1 . For values of $\alpha$ between 0 and 1, the Bush-Mosteller approach results in weights that decay exponentially over time (fig. 1b). The Bush-Mosteller approach does not allow for time lags.

We assess our new method using weight functions based 

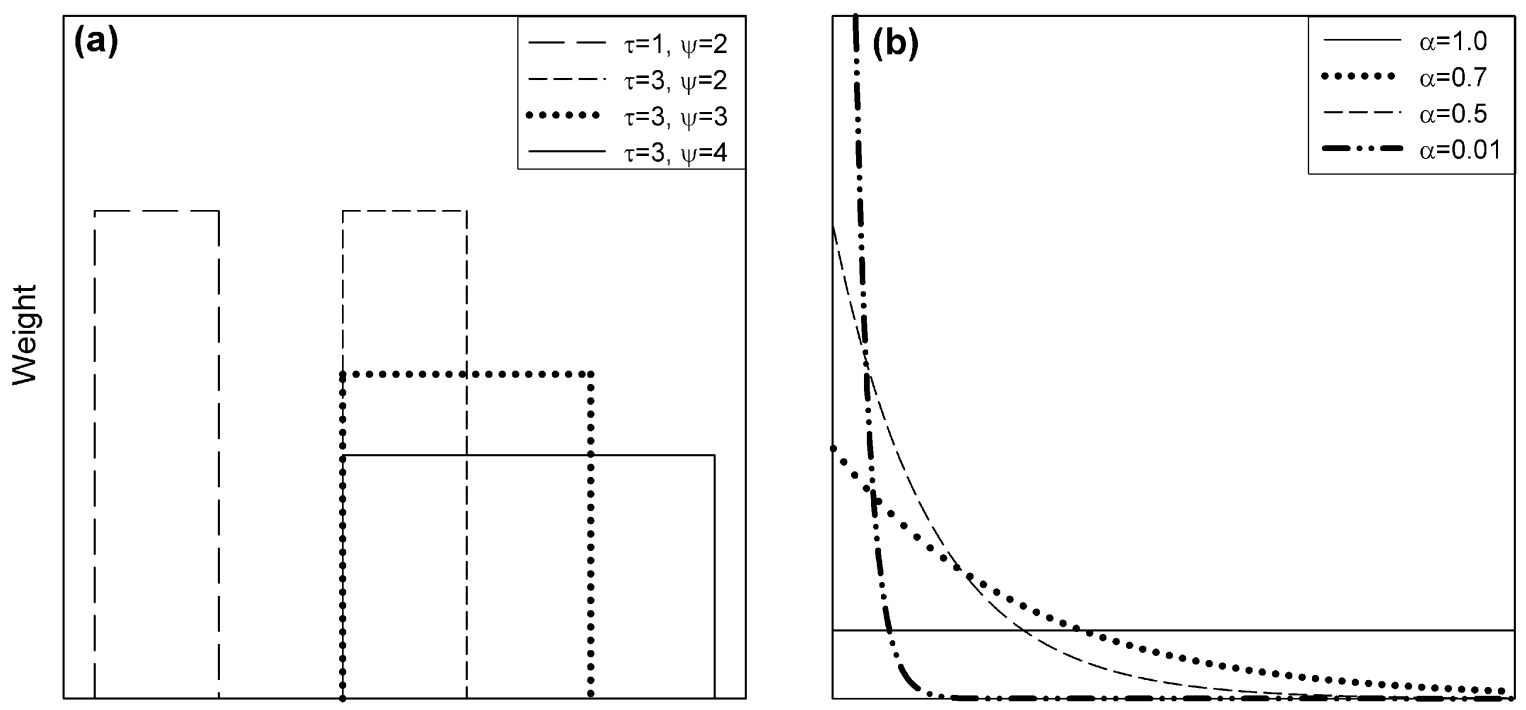

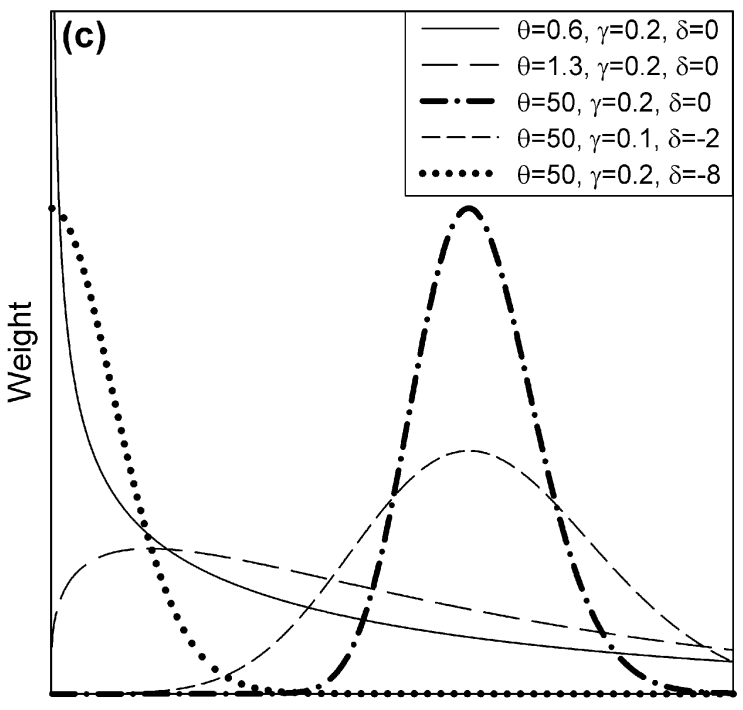

Time before trait expression

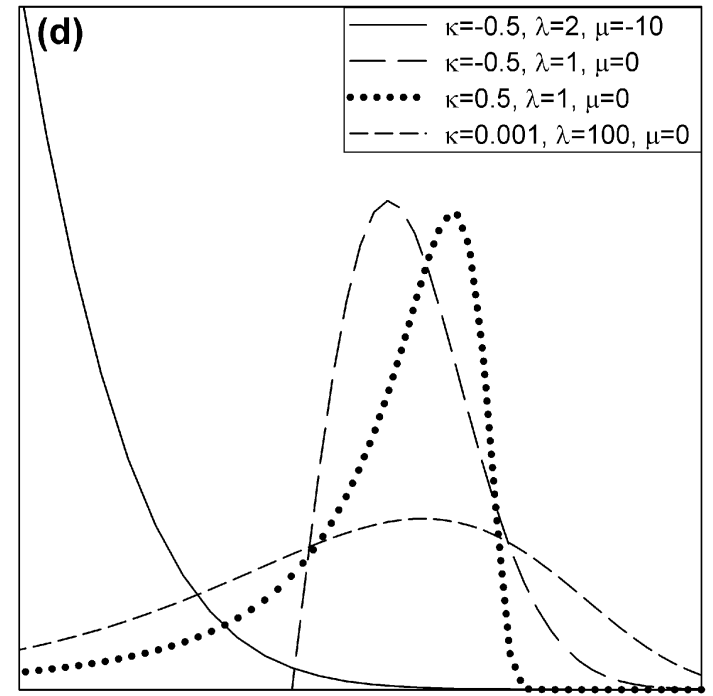

Time before trait expression

Figure 1: Graphical depiction of the type of weight functions available in (a) the sliding-window, $(b)$ the Bush-Mosteller, $(c)$ the Weibull, and $(d)$ the generalized extreme value method.

on two related probability distribution functions. The first is the well-known three-parameter Weibull function:

$$
w_{j}=\vartheta \gamma(j-\delta)^{\vartheta-1} \exp [-\gamma(j-\delta)]^{\vartheta},
$$

where $\vartheta>0, \gamma>0$, and $\delta \leq j$. The parameter $\vartheta$ determines the shape of the weight distribution, the scale parameter $\gamma$ determines the width of the distribution (i.e., window), and the location parameter $\delta$ determines the (sliding) location of the distribution (fig. 1c). The Weibull function allows for a wide range of weighting functions that include sliding windows lacking abrupt and discrete borders (fig.
$1 a$ vs. $1 c)$. In addition, the Weibull simplifies to an exponential distribution for $\vartheta=1$ and $\delta=0$, meaning that the Weibull weight function can produce the same type of responses as the Bush-Mosteller approach (fig. $1 b$ vs. 1c). The Bush-Mosteller model thus can be thought of as a particular case of the Weibull weight function. Finally, the Weibull function can also produce accelerating decays of past weather weights. Collectively, these features produce weighting functions that reflect various biological hypotheses about time lags and the way in which the importance of past weather changes over time. 
In principle, similar types of advantages can be obtained from using other distributions. The second weight function we tested, which allows for even greater flexibility, is the generalized extreme value (GEV) probability distribution function, which includes functions from the Fréchet, Gumbel, and reverse Weibull family (fig. 1d):

$$
w_{j}=\left\{\begin{array}{ll}
\lambda[1+\kappa \lambda(j-\mu)]^{-1-1 / \kappa} \exp \{-[1+\kappa \lambda(j-\mu)]\}^{-1 / \kappa} & \text { if } \kappa \neq 0 \\
\lambda \exp \{-\lambda(j-\mu)-\exp [-\lambda(j-\mu)]\} & \text { if } \kappa=0
\end{array},\right.
$$

where $\lambda>0$ and $1+\kappa \lambda(j-\mu)>0$. Similar to the Weibull, the GEV distribution has a shape parameter $\kappa$, a scale parameter $\lambda$, and a location parameter $\mu$. In contrast to the Weibull, the GEV distribution includes left-skewed, right-skewed, as well as fairly nonskewed functions, which allows for the comparison of competing hypotheses about how the importance of past weather for trait expression first increases and later decays over time. For $\kappa>0$ and using $-j$ instead of $j$, the GEV distribution simplifies to the three-parameter Weibull.

\section{Parameter Estimation}

We are interested in finding the parameter values of the weight function (e.g., $\kappa, \lambda$, and $\mu$ for the GEV) that result in the weather index that best describes the variation in the trait expression data. A practical hurdle is that these parameters cannot be estimated directly using conventional regression because our weather index (a predictor in the regression model) has become a function of model parameters itself, and so we are not dealing with a linear model. However, the likelihood function is known for any given set of values of $\kappa, \lambda$, and $\mu$, which means that optimization methods can be used to find the values of $\kappa$, $\lambda$, and $\mu$ that maximize the likelihood of the nonlinear regression model.

We used a quasi-Newton optimization method based on the limited memory variation of the Broyden-FletcherGoldfarb-Shanno algorithm, which specifically allows for upper- and lower-bound parameter constraints (Byrd et al. 1995). This optimization procedure can be easily implemented in the $\mathrm{R}$ language ( $\mathrm{R}$ Development Core Team 2009). An example of the required data format is given in appendix B, and we also provide an example of the source code (available in a zip file in the online edition of the American Naturalist).

\section{An Example: Timing of Prenuptial Molt in Superb Fairy Wrens}

We will illustrate our method using a 22-year data set on the timing of prenuptial molt in superb fairy wrens (Mal- urus cyaneus). Superb fairy wrens are sexually dimorphic birds in which the males can molt from a female-like brown eclipse plumage to a blue nuptial plumage. In contrast to the postbreeding molt that both sexes go through, the prenuptial molt is thought to be a sexually selected male signal. The timing of prenuptial molt predicts male extrapair mating success, which dominates reproductive success in this species (Cockburn et al. 2008).

Molt data were collected during year-round weekly censuses as part of a long-term study in Canberra, Australia (1988-2009, $n=1,307$ males of known age, 3,188 male years, 82,186 weeks at risk). Superb fairy wrens live in open habitat and are easy to approach. At least once a week, all males were carefully observed with binoculars to determine whether they had completed their prenuptial molt, as characterized by a full, glossy, light-blue crown, ear coverts, and mantle, as well as starkly contrasting dark lores, back, and throat (Mulder and Magrath 1994; Cockburn et al. 2008).

The time of completion of prenuptial molt is highly variable within and between individuals and can occur from March until December (fig. 2). Using sliding windows, Cockburn et al. (2008) showed that many males molt very early in winters that follow summers with high precipitation (November-March). In addition, Mulder and Magrath (1994) have shown that winters with high

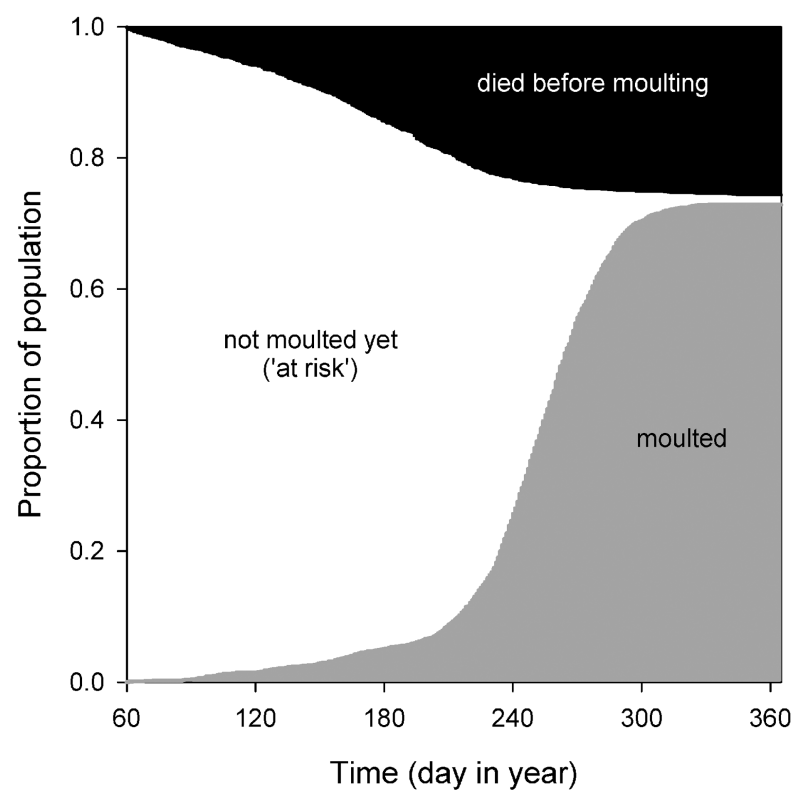

Figure 2: Changes during the year in the proportion of males in the population that (i) has not molted yet (individuals at risk; white area), (ii) has finished prenuptial molt (gray area), and (iii) has died before their molting event occurred (black area). Sample size is 3,188 male years during the period 1988-2009. 
Table 1: Model support for weather indices generated by different methods as determined by Akaike weights $\left(\mathrm{AIC}_{\mathrm{w}}=\exp \left(-\Delta \mathrm{AIC}_{\text {method }} / 2\right) / \sum\left[\exp \left(-\Delta \mathrm{AIC}_{\text {all }} / 2\right)\right]\right)$

\begin{tabular}{|c|c|c|c|c|c|c|c|}
\hline \multirow[b]{2}{*}{ Method } & \multirow[b]{2}{*}{ K } & \multicolumn{2}{|c|}{ Precipitation } & \multicolumn{2}{|c|}{$\begin{array}{l}\text { Minimum } \\
\text { temperature }\end{array}$} & \multicolumn{2}{|c|}{$\begin{array}{l}\text { Both variables } \\
\text { in model }\end{array}$} \\
\hline & & $\mathrm{AIC}_{\mathrm{w}}$ & $\Delta \mathrm{AIC}$ & $\mathrm{AIC}_{\mathrm{w}}$ & $\Delta \mathrm{AIC}$ & $\mathrm{AIC}_{\mathrm{w}}$ & $\Delta \mathrm{AIC}$ \\
\hline No weather index & 0 & $<.01$ & 360 & $<.01$ & 314 & $<.01$ & 444 \\
\hline Bush-Mosteller & 1 & $<.01$ & 192 & $<.01$ & 104 & $<.01$ & 132 \\
\hline Sliding window & 2 & .08 & 4.8 & $<.01$ & 30 & $<.01$ & 55 \\
\hline Weibull & 3 & $<.01$ & 20 & .25 & 2.2 & $<.01$ & 30 \\
\hline Generalized extreme value & 3 & .92 & 0 & .75 & 0 & .99 & 0 \\
\hline
\end{tabular}

Note: A model without weather index is included as a null model and contained 11 parameters to be estimated (see eq. [7]). $K$ equals the number of parameters used in each method's weight function (see eqq. [3]-[6]). AIC = Akaike Information Criterion.

average minimum temperature (May-September) result in years with an early mean start date of prenuptial molt. We will now apply our new method as well as existing methods to this data set (but with more years of data), while focusing on the same climatic variables (precipitation and minimum temperature).

\section{Climate Data}

Data on daily weather at Canberra airport (8 $\mathrm{km}$ east of the study area) were obtained from the Australian Bureau of Meteorology; missing precipitation and temperature values $(<0.1 \%$ of all values $)$ were linearly interpolated.

\section{Analyses of Phenological Traits}

Overwhelmingly, most studies analyze phenological traits in the same way as quantitative morphological, behavioral, or demographic traits: using standard linear regression models in which the Julian day of the timing event is the response variable (e.g., Estrella et al. 2007; Brommer et al. 2008; Charmantier et al. 2008; Husby et al. 2010). However, time-to-event models provide a much more appropriate framework with which to analyze phenological traits (e.g., Fox 1993; Gienapp et al. 2005). Time-to-event models-such as Cox regression, proportional hazard models, and accelerated failure time models-have at least two crucial advantages. First, time-to-event models allow for the inclusion of individuals that died or disappeared before the trait of interest was expressed, which can be quite a substantial fraction (fig. 2). Standard linear regression models typically exclude individuals with missing response data, which can lead to biased results because such data may not be missing completely at random (Nakagawa and Freckleton 2008), which is doubly important where selection is involved (Hadfield 2008).

Second, in standard linear regression models, the critical time window is the same for all individuals, which may be unrealistic for phenological events that can occur over long periods. For example, March temperatures may be likely to affect molting rates in April but unlikely to affect molting rates in November. In extended Cox models (Kleinbaum and Klein 2005), time-dependent climatic covariates can be used that allow the critical time window to vary over time (Gienapp et al. 2005), such that molting rates throughout the year may depend on the temperature in, for example, the preceding month.

In our analyses we used the following extended Cox model:

$$
\begin{gathered}
\operatorname{hazard}\left(t, I_{\text {weather }} \text {, age, frailty }\right)= \\
\left.h_{0}(t) \exp \left[\beta_{1} I_{\text {weather }}(t)+\text { factor(age }\right)+\text { frailty }_{\text {individual }}\right] .
\end{gathered}
$$

In this model the molting hazard rate depends on a timedependent baseline hazard $h_{0}(t)$ and several time-dependent and time-independent predictors. The weather index $I$ depends on the precipitation or minimum temperature data as well as the chosen weight functions and their associated parameters (eqq. [2]-[6]). We included age as a factor $(1,2,3,4,5,6,7,8+)$, since molting behavior is age dependent in a nonlinear way (Mulder and Magrath 1994). Moreover, a gamma-distributed shared frailty term was included to account for individual heterogeneity in molting hazard (nonindependency; individual males molted in multiple years). The support by our data for each fitted method (sliding window, Bush-Mosteller, Weibull weights, and GEV weights) was quantified by the Akaike Information Criterion (Burnham and Anderson 2002).

\section{Results}

\section{Comparison between Methods}

The GEV-weight method received the highest relative model support of the models we fitted to generate weather 

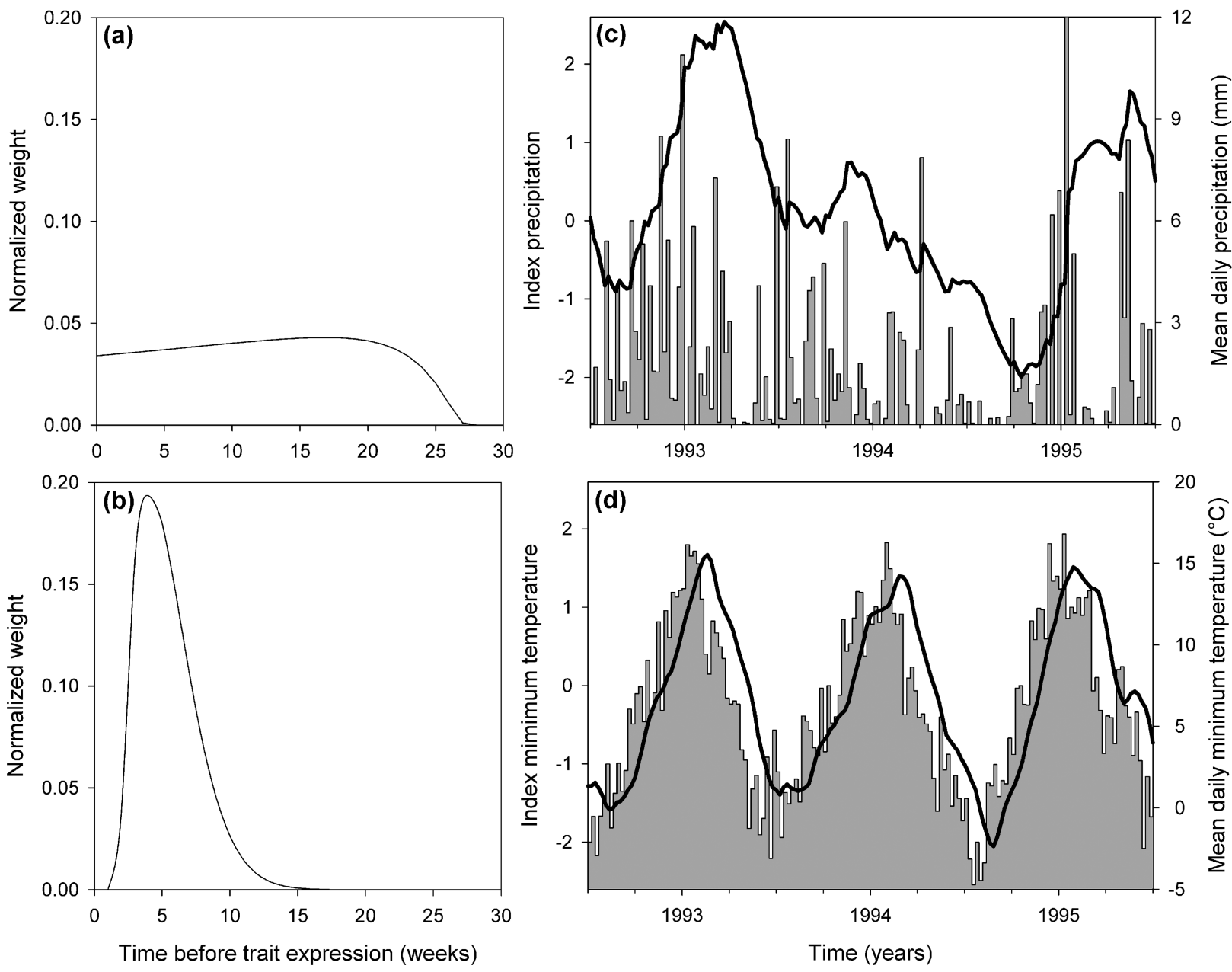

Figure 3: Weight function of $(a)$ the precipitation index and $(b)$ the minimum temperature index that were best supported by the data (generalized extreme value method; see table 1). In $c$ and $d$, the corresponding precipitation and minimum temperature index are illustrated for 3 years, depicted by the solid lines (left $Y$-axes), while the gray bars represent the underlying weather data (right $Y$-axes). Note that weights are normalized such that they sum to 1 , and weather indices are standardized to 0 mean and standard deviation 1 over the study period.

indices, for both the precipitation and minimum temperature index (table 1). The Weibull-weight method produced a minimum temperature index that also received some model support, but the precipitation index generated by this method was far less well supported. Conversely, the sliding-window approach produced a precipitation index that also received some support, while the minimum temperature index generated by this method was far less well supported. The Bush-Mosteller approach produced precipitation and minimum temperature indices that both received much less model support.

When we entered both weather indices simultaneously to the model, the GEV-weight method was again by far best supported by the data (table 1). A model including both weather indices generated by the GEV-weight method explained a total of $12.1 \%$ of the weekly variation in molting rates of superb fairy wrens, which was more than for any of the other methods (Nagelkerke's $R_{\text {Bush-Mosteller }}^{2}=$ $\left.8.5 \%, R_{\text {sliding-window }}^{2}=10.5 \%, R_{\text {Weibull }}^{2}=11.4 \%\right)$.

\section{Best-Fitting Critical Time Period}

The best supported precipitation index (effect size $\beta=$ $0.30 \pm 0.04 \mathrm{SE}$ ) suggested that molting hazard primarily depends on the precipitation in the preceding 25 weeks, without much variation in contribution among time intervals across this period (fig. $3 a, 3 c$ ). By contrast, the best supported minimum temperature index (effect size $\beta=$ $0.96 \pm 0.12 \mathrm{SE}$ ) suggested that molting hazard primarily depends on the preceding 2-15 weeks, with the temper- 
ature in the two most recent weeks (week 0 and week 1) not contributing to this index (fig. $3 b, 3 d$ ). Furthermore, days in the preceding 3-7 weeks contributed most strongly to this temperature index, suggesting that the effect of temperature on molting rates gradually builds up in the 8 weeks before (weeks $8-15$ ).

\section{Discussion \\ Critical Climatic Periods for Molting Fairy Wrens}

The critical climatic period for the timing of molting in superb fairy wrens was strikingly different for the two weather variables considered (fig. $3 a$ vs. $3 b$ ). Weekly molting rates were affected by precipitation over a long period, while the effects of temperature were much more short term. Molting is energetically demanding, and climate effects could thus act via both food intake and energy expenditure (Mulder and Magrath 1994 and references therein). Possibly, the long critical precipitation period of these insectivorous birds reflects delayed effects of rainfall on vegetation and thereby insect growth and abundance, while the effects of minimum temperature might act more directly on the energy expenditure and thus body condition of birds. A logical next step would be to include data on insect phenology and abundance into the analyses, which could be analyzed in ways similar to those used for climatic variables.

How time-dependent weather indices vary within and among years may provide further insights into the role of different weather variables. The annual pattern of the bestsupported temperature index was very similar across years (fig. 3d), suggesting that the strong and predictable seasonality of temperature may explain primarily intraannual variation in molting rates. By contrast, the annual pattern of the best-supported precipitation index varied strongly across years (fig. $3 c$ ), suggesting that precipitation may explain much of the interannual variation in molting rates.

\section{Advantages of the New Method}

Our method produced weather indices that explained more of the variation in trait expression in our example data set than existing methods. Our new method is likely to generally perform at least as well as-and usually better than-conventional associative methods because it combines the strong points of the sliding-window and BushMosteller approaches into a single method. On top of that, the new method offers additional flexibility because of the wide variety of weight functions that can be used. This flexibility comes at little extra cost in terms of model com- plexity, as our method requires only one and two additional parameters to be estimated than in the BushMosteller and sliding-window approaches, respectively. Finally, our new method can be applied to any timescale, including the analyses of multiyear time lags of climate effects on demographic traits (e.g., Leaper et al. 2006).

Our intuitive method should thus provide biologists with a better tool to distinguish between competing hypotheses about critical climatic windows and to identify the causes of trait response to environmental variability. Identifying the correct critical periods will be especially important if climate change affects the weather at various times of the year differentially (Easterling et al. 1997). In such a scenario, misidentified critical periods could be poor predictors of the future climatic change relevant for trait expression, which may lead to unreliable projections of future trait and population dynamics (above all for studies on phenological synchrony in food webs).

\section{Time-Dependent versus Fixed Critical Periods}

In our analyses of a phenological trait we used a weather index that varied over time: molting rate at any given point in time depended on the precipitation in the preceding 25 weeks (fig. 3a). This approach is fundamentally different from the vast majority of studies that use a fixed period, in which the timing of trait expression always depends on, for example, January-March precipitation (e.g., Estrella et al. 2007; Brommer et al. 2008; Charmantier et al. 2008; Cockburn et al. 2008; Husby et al. 2010; but see Gienapp et al. 2005, 2010). Clearly, if the timing of trait expression varies over a long period or if the climatic time lag is short, using a fixed critical period is unlikely to produce predictive weather indices. For the same reason, a fixed critical period will often be less suitable for the demographic trait survival, and the climate dependency of mortality should preferentially also be analyzed using time-dependent weather indices (Hallett et al. 2004).

The common use of a fixed critical period has also been invoked as a reason why many studies find that large-scale climatic indices-such as the North Atlantic oscillation, El Niño-Southern oscillation, and Indian Ocean dipole index-often predict (demographic) trait expression better than local weather variables (Hallett et al. 2004; Stenseth and Mysterud 2005). Large-scale climatic indices are often a fairly good predictor of whether, for example, a severe cold spell is coming this winter or not (Shabbar and Bonsal 2003). However, because such a cold spell can occur in different months in different years, a fixed critical period typically does not always capture the temporal variability in temperature well. Time-dependent weather indices are therefore likely to be a key feature of many "weather packages" (sensu Stenseth and Mysterud 2005). 


\section{Acknowledgments}

We thank everyone who contributed to data collection, especially H. Osmond. M. Hall, R. Altwegg, and an anonymous reviewer provided helpful comments that improved the manuscript. M.v.d.P. was supported by an Australian
Postdoctoral Fellowship of the Australian Research Council (DP1092565). Data were collected under a series of Discovery Grants from the Australian Research Council. Permits were provided by the Australian National University Animal Experimentation Ethics Committee and the Australian National Botanic Gardens.

\section{APPENDIX A}

\section{Rewriting the Bush-Mosteller Equation to a Weight Function}

The Bush-Mosteller equation is as follows:

$$
I_{t}=(1-\alpha) c_{t}+\alpha I_{t-1}
$$

Writing out the recursive equation for two time steps shows that

$$
I_{t}=(1-\alpha) \mathcal{c}_{t}+\alpha\left\{(1-\alpha) \mathcal{c}_{t-1}+\alpha\left[(1-\alpha) c_{t-2}+\alpha I_{t-3}\right]\right\}
$$

Or, more generally,

$$
I_{t}=(1-\alpha)\left(c_{t}+\alpha c_{t-1}+\alpha^{2} c_{t-2}+\alpha^{3} c_{t-3}+\ldots+\alpha^{p-1} c_{t-p-1}\right)+\alpha^{p} I_{t-p} .
$$

This can be written more compactly as

$$
I_{t}=(1-\alpha) \sum_{j=0}^{j=p-1}\left(\alpha^{j} c_{t-j}\right)+\alpha^{p} I_{t-p} .
$$

If we assume the period of interest $p$ is sufficiently large, then the contribution of the most distant time interval is negligible $\left(\alpha^{p} I_{t-p} \approx 0\right)$. In addition, the term $(1-\alpha)$ is a redundant scalar, as it is multiplied to all time steps. Consequently, equation (A4) simplifies to:

$$
I_{t} \propto \sum_{j=0}^{j=p} \alpha^{j} c_{t-j}
$$

Comparing equation (A5) with equation (2) then shows that for the Bush-Mosteller $w_{j}=\alpha^{j}$.

\section{APPENDIX B}

\section{Example of Data Format and R Source Code to Find the Weight Parameters That Maximize the Likelihood Function}

We assume there are two input-data files: one file with the trait expression data (trait.txt) and one file with weather data (weather.txt). The trait expression data in our example is structured for use in an extended Cox model (eq. [7]), as our example deals with a phenological trait (time of prenuptial molting). However, our extended Cox model can be replaced by any generalized linear (mixed) model when analyzing quantitative traits, such as many morphological, behavioral, or life-history traits. A view of the data structure of both data files is given in tables B1 and B2. The example data set used in this article is available at http://datadryad.org/handle/10255/dryad.8277. A zip file in the online edition contains the file OptimWeight.r, which gives an example of the R source code (R Development Core Team 2009) that can be used to find the parameters of the weight function that produce the weather index that best explains the variation in trait expression for the Weibull and GEV method. 
Table B1: View of the data structure in the input-data file trait.txt

\begin{tabular}{lcccc}
\hline $\begin{array}{l}\text { Individual } \\
\text { ID }\end{array}$ & Year & $\begin{array}{c}\text { Time } \\
\text { (e.g., weeks, days) }\end{array}$ & $\begin{array}{c}\text { Event } \\
\text { (molted) }\end{array}$ & $\begin{array}{c}\text { Age } \\
\text { (years) }\end{array}$ \\
\hline 1 & 1999 & 1 & 0 & 3 \\
1 & 1999 & 2 & 0 & 3 \\
1 & 1999 & 3 & 0 & 3 \\
1 & 1999 & 4 & 1 & 3 \\
1 & 2000 & 1 & 0 & 4 \\
1 & 2000 & 2 & 1 & 4 \\
2 & 1992 & 1 & 0 & 1 \\
2 & 1992 & 2 & 0 & 1 \\
3 & 1988 & 1 & 0 & 1 \\
3 & 1988 & 2 & 0 & 1 \\
3 & 1988 & 3 & 0 & 1 \\
3 & 1988 & 4 & 1 & 1 \\
3 & 1989 & 1 & 0 & 2 \\
3 & 1989 & 2 & 1 & 2 \\
\hline
\end{tabular}

Note: Individual 2 was censored (died before molting) at time $=2$, and thus event $=0$ there. Age is a time-independent predictor used in the super fairy wren example data set.

Table B2: View of the data structure in the input-data file weather.txt

\begin{tabular}{lcc}
\hline Year & $\begin{array}{c}\text { Time } \\
\text { (e.g., weeks, days) }\end{array}$ & $\begin{array}{c}\text { Precipitation } \\
\text { (e.g., } \mathrm{mm} / \text { week, mm/day) }\end{array}$ \\
\hline 1987 & 1 & 2.1 \\
1987 & 2 & 0 \\
1987 & 3 & 0 \\
1987 & 4 & 1.8 \\
1987 & $\ldots$. & $\ldots$ \\
1987 & 52 & 4.2 \\
1988 & 1 & 16.5 \\
1988 & 2 & 5.6 \\
1988 & $\ldots$ & $\ldots$ \\
1988 & 52 & 1.1 \\
$\ldots$. & $\ldots$ & $\ldots$ \\
2009 & 51 & .2 \\
2009 & 52 & 0 \\
\hline
\end{tabular}

Note: The weather data are sorted on time (years then weeks), and we assume there are no missing values.

\section{Literature Cited}

Bauer, S., P. Gienapp, and J. Madsen. 2008. The relevance of envi ronmental conditions for departure decision changes en route in migrating geese. Ecology 89:1953-1960.

$\rightarrow$ Brommer, J. E., K. Rattiste, and A. J. Wilson. 2008. Exploring plas ticity in the wild: laying date-temperature reaction norms in the common gull Larus canus. Proceedings of the Royal Society B: Biological Sciences 275:687-693.

Burnham, K. P., and D. R. Anderson. 2002. Model selection an multimodel inference. Springer, New York.
Bush, R. R., and F. Mosteller. 1955. Stochastic models for learning. Wiley, New York.

$\rightarrow$ Byrd, R. H., P. Lu, and J. Nocedal. 1995. A limited memory algorithm for bound constrained optimization. SIAM Journal on Scientific and Statistical Computing 16:1190-1208.

$\rightarrow$ Charmantier, A., R. H. McCleery, L. R. Cole, C. Perrins, L. E. B. Kruuk, and B. C. Sheldon. 2008. Adaptive phenotypic plasticity in response to climate change in a wild bird population. Science 320:800-803.

$\rightarrow$ Cockburn, A., H. Osmond, and M. C. Double. 2008. Swingin' in the rain: condition dependence and sexual selection in a capricious 
world. Proceedings of the Royal Society B: Biological Sciences 275: 605-612.

$\rightarrow$ Easterling, D. R., B. Horton, P. D. Jones, T. C. Peterson, T. R. Karl $\rightarrow$ D. E. Parker, M. J. Salinger, et al. 1997. Maximum and minimum temperature trends for the globe. Science 277:364-367.

$\rightarrow$ Estrella, N., T. H. Sparks, and A. Menzel. 2007. Trends and temper $\rightarrow$ Nak ature response in the phenology of crops in Germany. Global Change Biology 13:1737-1747.

$\rightarrow$ Fitter, A. H., R. S. R. Fitter, I. T. B. Harris, and M. H. Williamson $\rightarrow$ 1995. Relationship between first flowering date and temperature in the flora of a locality in central England. Functional Ecology 9: $55-60$.

Fox, G. A. 1993. Failure time analysis: emergence, flowering, survivorship, and other waiting times. Pages 253-289 in S. M. Scheiner and J. Gurevitch, eds. Design and analysis of ecological experi $\rightarrow$ ments. Chapman \& Hall, New York.

$\rightarrow$ Gienapp, P., L. Hemerik, and M. E. Visser. 2005. A new statistical tool to predict phenology under climate change scenarios. Globa $\rightarrow$ Change Biology 11:600-606.

Gienapp, P., R. A. Vaisanen, and J. E. Brommer. 2010. Latitudina $\rightarrow$ Spar variation in breeding time reaction norms in a passerine bird. Journal of Animal Ecology 79:836-842.

$\rightarrow$ Hadfield, J. D. 2008. Estimating evolutionary parameters when vi $\rightarrow$ ability selection is operating. Proceedings of the Royal Society B: Biological Sciences 275:723-734.

$\rightarrow$ Hallett, T. B., T. Coulson, J. G. Pilkington, T. H. Clutton-Brock, J $\rightarrow$ M. Pemberton, and B. T. Grenfell. 2004. Why large-scale climate indices seem to predict ecological processes better than local weather. Nature 430:71-75.

Husby, A., D. H. Nussey, M. E. Visser, A. J. Wilson, B. C. Sheldon, and L. E. B. Kruuk. 2010. Contrasting patterns of phenotypic plasticity in reproductive traits in two great tit (Parus major) populations. Evolution 68:2221-2237.

$\rightarrow$ Izquierdo, J., J. L. Gonzalez-Andujar, F. Bastida, J. A. Lezaun, and M. J. Sanchez del Arco. 2009. A thermal time model to predict corn poppy (Papaver rhoeas) emergence in cereal fields. Weed Science 57:660-664.

Kleinbaum, D. G., and M. Klein. 2005. Survival analysis: a selflearning text. 2nd ed. Springer, New York.

$\rightarrow$ Leaper, R., J. Cooke, P. Trathan, K. Reid, V. Rowntree, and R. Payne.
2006. Global climate drives southern right whale (Eubalaena australis) population dynamics. Biology Letters 2:289-292.

Mulder, R. A., and M. J. L. Magrath. 1994. Timing of prenuptial molt as a sexually selected indicator of male quality in superb fairy-wrens. Behavioral Ecology 5:393-400.

Nakagawa, S., and R. P. Freckleton. 2008. Missing inaction: the dangers of ignoring missing data. Trends in Ecology \& Evolution 23: 592-596.

Ozgul, A., S. Tuljapurkar, T. G. Benton, J. M. Pemberton, T. H. Clutton-Brock, and T. Coulson. 2009. The dynamics of phenotypic change and the shrinking sheep of St. Kilda. Science 325:464-467.

R Development Core Team. 2009. R: a language and environment for statistical computing. R Foundation for Statistical Computing, Vienna.

Roberts, A. 2008. Exploring relationships between phenological and weather data using smoothing. International Journal of Biometeorology 52:463-470.

Shabbar, A., and B. Bonsal. 2003. An assessment of changes in winter cold and warm spells over Canada. Natural Hazards 29:173-188.

Sparks, T. H., and P. D. Carey. 1995. The response of species to climate over two centuries: an analysis of the Marsham phenological record, 1736-1947. Journal of Ecology 83:321-329.

Stenseth, N. C., and A. Mysterud. 2005. Weather packages: finding the right scale and composition of climate in ecology. Journal of Animal Ecology 74:1195-1198.

Trudgill, D. L., A. Honek, D. Li, and N. M. Van Straalen. 2005. Thermal time-concepts and utility. Annals of Applied Biology 146:1-14.

$\rightarrow$ van de Pol, M., Y. Vindenes, B. E. Sæther, S. Engen, B. J. Ens, K. Oosterbeek, and J. M. Tinbergen. 2010. Effects of climate change and variability on population dynamics in a long-lived shorebird. Ecology 91:1192-1204.

$\rightarrow$ Visser, M. E. 2008. Keeping up with a warming world: assessing the rate of adaptation to climate change. Proceedings of the Royal Society B: Biological Sciences 275:649-659.

Associate Editor: Jean-Michel Gaillard Editor: Mark A. McPeek
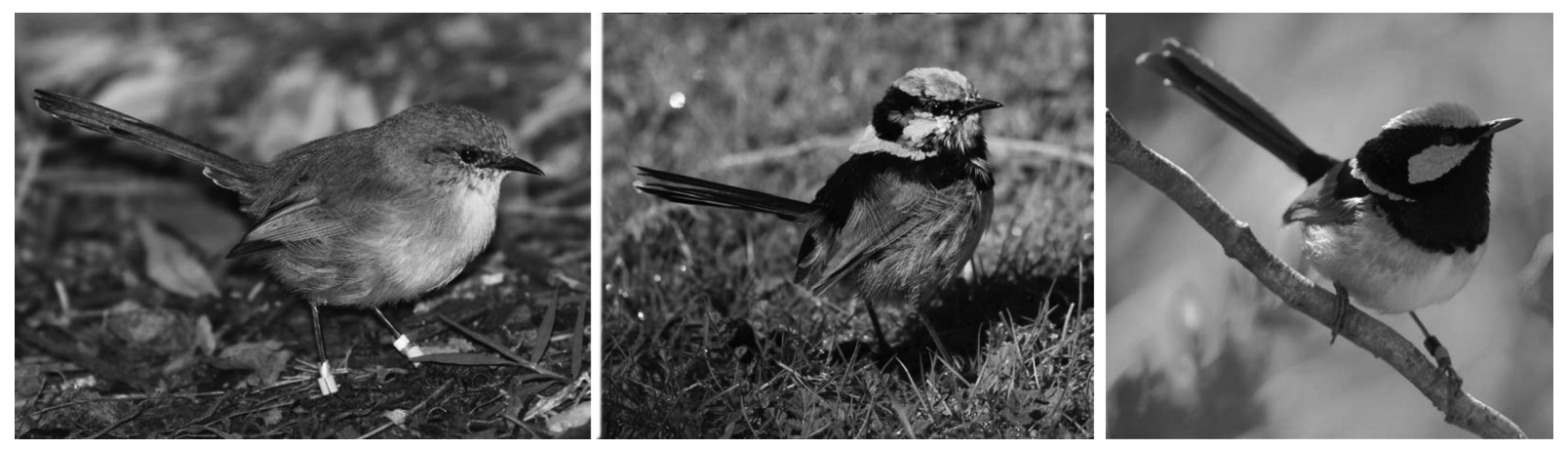

The timing of the transition from a brown eclipse plumage (left), via a partly molted plumage (center), to a full nuptial plumage (right) is dependent on a long rainfall window and a short temperature window in male superb fairy wrens living in the National Botanic Gardens of Canberra. Photographs by Geoffrey Dabb (all rights reserved). 